\title{
Caractérisation des fonds marins par acoustique : état de l'art et perspectives
}

\author{
X. LURTON
}

IFREMER, Centre de Brest, Laboratoire d'Acoustique Sous-Marine, DITI/SM/ASM, BP. 70, 29280 Plouzane, France

\begin{abstract}
Acoustical characterization of the sea-floor may be performed in different ways depending of the type of system to be used. Several techniques are presented here for sea-bed identification using mono-beam echo-sounders (time signal analysis, second echo amplitude) and side scan sonars (spectral analysis, image texture). New possibilities are provided by recent multi-beam echo-sounders, performing both bathymetry measurements and imagery. The previous techniques could be used for these systems, together with direct measurements of reflectivity angular dependence. Finally are presented here some results obtained in deep water with a low frequency multibeam sounder.
\end{abstract}

\section{INTRODUCTION}

Les techniques rapides d'identification de la nature du fond de la mer ont de nombreuses applications potentielles, et les enjeux correspondants peuvent être d'importance. Scientifiques, d'abord: la géologie marine a besoin d'outils permettant d'effectuer une première analyse de la structure d'une zone sédimentaire que l'on peut ensuite affiner par d'autres méthodes. Dans le domaine de l'ingéniérie sousmarine, lidentification de la nature du fond est de première importance pour, par exemple, la pose de câbles de télécommunications. Dans le domaine de la Défense, la connaissance de la nature du fond sur les zones opérationnelles est un des éléments clés de la maitrise des conditions de propagation acoustique et est donc importante pour les opérations de guerre sous-marine; on notera par ailleurs que le relevé des cartes de navigation, qui mentionnent des indications de la nature du fond, est en France de la responsabilité de la Marine Nationale. Pour la pêche, la connaissance instantanée de la nature du fond est un élément important pour la prospection, du fait de la corrélation entre cette nature et les espèces de poissons recontrés. Enfin l'intérêt de l'identification est évident pour l'activité minière offshore actuelle (dragage de sédiments) ou potentielle (collecte de nodules polymétalliques).

Les systèmes acoustiques peuvent dans une large mesure répondre à de tels besoins. Ils sont utilisés depuis longtemps pour mesurer la hauteur d'eau (ou sonde) sous un navire, pour détecter des obstacles posés sur le fond et pour relever des images acoustiques des fonds marins; mais on ne s'est intéressé que relativement récemment à l'utilisation des signaux réverbérés pour caractériser la nature du fond de la mer.

Quel type de caractérisation des fonds marins un système acoustique de bathymétrie ou d'imagerie peut-il prétendre effectuer? Il est clair que les potentialités restent modestes face aux exigences des géologues; ceux-ci classifient les sédiments en terme de granulométrie, et de composition minéralogique, deux grandeurs auxquelles l'analyse acoustique n'a pas accès directement, au moins dans les gammes de fréquences des systèmes usuels (de 3.5 à $400 \mathrm{kHz}$ ). Les caractéristiques du fond intervenant dans le signal acoustique rétrodiffusé, et donc éventuellement analysables à partir de ces signaux, sont limpédance acoustique (densité, célérité(s), atténuation), son évolution et la présence d'inhomogénéités dans les strates superficielles, et les caractéristiques du micro-relief de l'interface (hauteurs, pentes, longueurs de corrélation). Encore l'analyse acoustique n'aura-t-elle accès qu'à un mélange des contributions de ces différents facteurs, qui ne seront dissociables que dans des conditions de mesures particulières; mais il faut aussi remarquer que ces diverses caractéristiques sont fortement corrélées entre elles (par exemple les 
sédiments à forte impédance sont susceptibles de supporter de fortes valeurs de micro-relief). Le système acoustique aura finalement accès à un type de description globale faisant intervenir ces diverses composantes, et traduisant plutôt des propriétés mécaniques élémentaires (dureté-relief) qu'une réalité géologique bien identifiable. Les différents systèmes proposés convergent en général vers des classifications à l'intérieur de quelques catégories assez qualitatives (typiquement 5 à 10), par exemple: argile, vase, sable fin, sable grossier, gravier, boulets, rochers. Il est à noter qu'une telle classification peut néanmoins très bien suffire à de nombreuses applications, et que même le géologue peut y trouver son compte dans le cadre limité d'une première approche de la structure d'une zone.

\section{RETRODIFFUSION DES ONDES ACOUSTIQUES PAR LES FONDS MARINS}

Le signal rétrodiffusé par le fond de l'océan est dû à plusieurs composantes:

- réflexion spéculaire cohérente à la verticale, dont l'importance réelle est souvent faible du fait du faible rapport longueur d'onde/hauteur de relief usuellement rencontré, mais qui peut être notable pour des signaux sondeurs basse fréquence sur des sédiments à faible nugosité,

- rétrodiffusion par le micro-relief de l'interface, aux incidences proches de la verticale, que l'on peut le plus souvent décrire théoriquement de manière pertinente par la méthode du plan tangent [1]. Maximale près de la verticale, cette contribution diminue très rapidement quand l'angle d'incidence augmente, d'autant que la variance des pentes du relief est faible.

- rétrodiffusion par les inhomogénéités du sédiment, de l'onde ayant traversé l'interface. Cette contribution dépend faiblement de l'angle d'incidence, tant que celui-ci est inférieur à l'angle critique de l'interface.

De manière générale l'indice de rétrodiffusion par le fond décroit très rapidement depuis les faibles angles d'incidence, et se stabilise au delà de 10 à $30^{\circ}$; on donne en fig. 1 un exemple de modèle théorique d'indice de réverbération de fond, synthétisant les modèles de Brekhovskikh et Lysanov [1] pour les effets d'interface et de volume et pour plusieurs types de fonds.

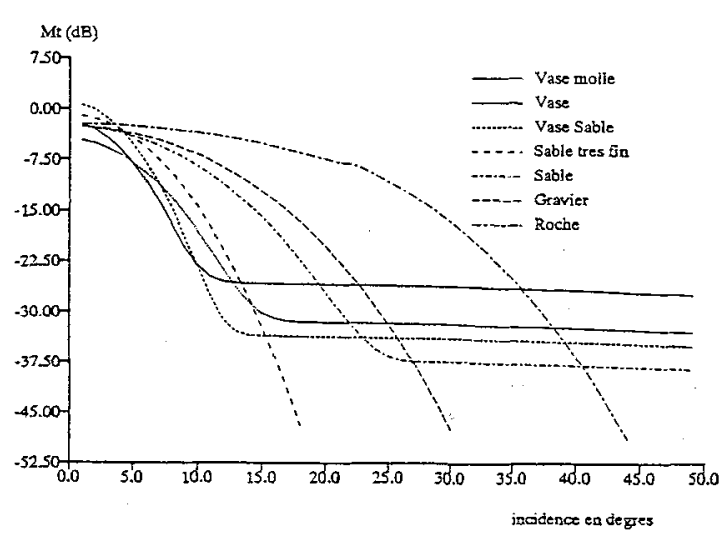

Fig. 1. Exemple d'indice de rétrodiffusion calculé par les modèles de Brekhovskikh et Lysanov [1]

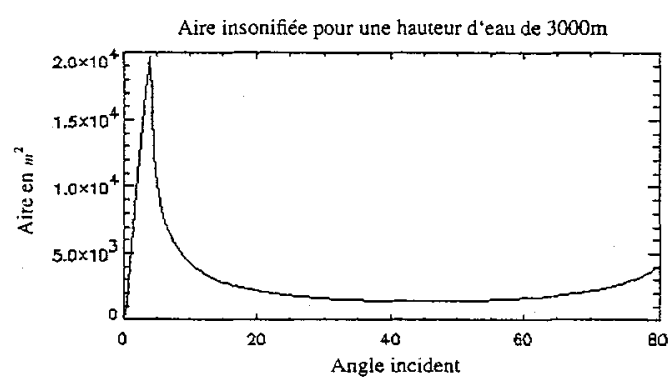

Fig.2. Exemple de calcul de l'évolution de la section insonifiée instantanée avec l'angle d'incidence, pour un sondeur multifaisceaux

L'écho de fond est dû à l'interaction d'un signal de courte durée émis à l'intérieur du lobe de directivité (émission et réception) du système avec le fond réverbérant. Il est maximal près de la verticale, et décroît aux plus fortes incidences. Ce comportement résulte à la fois de la dépendance angulaire de lindice de rétrodiffusion (fig.1), et de l'effet géométrique de construction de la surface de fond insonifiée localement (fig.2) limitée soit par l'ouverture. Il en résulte un niveau très inhomogène des signaux acoustiques reçus, avec par exemple pour effet une bande centrale très marquée sur les images acoustiques obtenues. Les dynamiques peuvent atteindre 40 à $60 \mathrm{~dB}$. 


\section{LES INSTRUMENTS DE LA CARTOGRAPHIE ACOUSTIQUE SOUS-MARINE: SONDEURS ET SONARS}

Un certain nombre de travaux ont été entrepris sur la caractérisation de la rétrodiffusion par les fonds, et leur identification à partir de données fournies par des dispositifs expérimentaux prototypes spécialement conçus à cet usage; par exemple dans [2,3] sont présentés des résultats obtenus avec une antenne paramétrique utilisée pour divers signaux, fréquences et incidences. De telles approches permettent une investigation systématique et fine de la nature des phénomènes physiques et éventuellement des potentialités de diverses méthodes. Nous ne reprendrons pas ici les résultats de ce type d'études, nous restreignant à l'analyse des applications faisant intervenir des systèmes couramment opérationnels.

Les appareillages les plus couramment utilisés pour la mesure de la bathymétrie (hauteur d'eau) sont les sondeurs monofaisceaux; ce type d'appareil fait maintenant partie de la panoplie normale des systèmes de navigation maritime, et est extrêmement répandu. Ces dispositifs utilisent le plus couramment un faisceau vertical d'ouverture de l'ordre d'une dizaine de degrés, avec des impulsions de durée comprise entre 0,1 et $1 \mathrm{~ms}$; les fréquences utilisées vont de $12 \mathrm{kHz}$ (pour les versions grands fonds) à $200 \mathrm{kHz}$. Des systèmes très semblables sont utilisés pour la pêche; en plus de l'information de l'écho de fond, le sondeur doit alors être capable de traiter et gérer les échos issus du "volume" de l'océan (poissons isolés ou en bancs).

Relativement récemment ont été introduits dans le domaine de l'océanographie les sondeurs multifaisceaux de bathymétrie. Ces systèmes comportent un éventail de faisceaux élémentaires très directifs, qui permettent de récupérer, à partir d'un seul signal émis, plusieurs échos du fond disposés sur un ligne transversale à la route du navire; on relève ainsi non plus un tracé linéaire du profil de fond à l'aplomb de la route du navire, mais le relief sur une largeur notable, d'où un formidable gain d'efficacité. De performances assez modestes au départ (ouverture de $20^{\circ}$ de part et d'autre de la verticale avec le "Sea-Beam" installé en 1977 sur le "Jean Charcot") les sondeurs multifaisceaux ont effectué de notables progrès de performances (ouverture de $75^{\circ}$ de part et d'autre de la verticale avec un pas de $1^{\circ}$ pour le Simrad EM12D installé en 1989 sur "L'Atalante"). Ces systèmes constituent de formidables outils d'investigation des fonds sousmarins, et dont toutes les potentialités ne sont pas encore exploitées aujourd'hui.

Les sonars latéraux de cartographie sous-marine sont destinés à relever des "images acoustiques" des fonds marins à partir d'un signal très bref (de l'ordre de $0,1 \mathrm{~ms}$ ) émis quasi-horizontalement dans un faisceau très étroit horizontalement (de l'ordre de $1^{\circ}$ ) et réverbéré par le fond; le niveau rétrodiffusé traduit la réflectivité du fond et la présence d'obstacles naturels ou artificiels. Habituellement installés sur un "poisson" remorqué près du fond, ces systèmes sont couramment utilisés par les géologues pour étudier la structure superficielle des fonds marins, surtout en petits fonds pour lesquels de nombreux appareillages légers et performants existent et sont couramment mis en oeuvre, mais aussi aux grandes profondeurs avec par exemple le SAR (Système Acoustique Remorqué) de l'IFREMER, sonar latéral à grande portée horizontale utilisable à $6000 \mathrm{~m}$ d'immersion.

Il est à noter que les deux types de systèmes (sondeurs et sonars) tendent de plus en plus à fusionner en des systèmes multifonctions. Ainsi les sondeurs multifaisceaux Simrad EM12 et EM1000 sont-ils capables d'assurer à la fois une fonction bathymétrie et le relevé d'images analogues à celles d'un sonar latéral, mais avec une couverture possible bien plus importante, qui les rendent spécialement performants et prometteurs pour les applications aux études de géosciences marines sur des zones étendues [4].

\section{UTLLISATION DES SONDEURS MONOFAISCEAUX POUR L'IDENTIFICATION DES FONDS}

L'utilisation des signaux issus de sondeurs monofaisceaux pour lidentification du fond de la mer est sans doute contemporaine de l'apparition de ces systèmes: tout utilisateur un peu expérimenté de sondeur est capable de se faire une idée du type de fond à partir de la visualisation graphique de l'écho de fond, plus ou moins intense et étalé. Toutefois cette identification reste limitée, pour un utilisateur, à son sondeur usuel, et à un petit nombre de configurations couramment rencontrées. En fait l'utilisation automatisée du sondeur pour une détermination objective de la nature du fond pose un certain nombre de problèmes, et n'a donné lieu qu'à peu de réalisations effectives.

Le niveau moyen d'énergie du signal rétrodiffusé par le fond pouriait à première vue sembler la principale information exploitable pour un sondeur monofaisceau; il n'est cependant qu'assez modérément informatif. L'amplitude moyenne de l'écho obtenu ne dépend que grossièrement de la nature du fond, car 
elle fait intervenir à la fois le contraste d'impédance, la rugosité de l'interface et les inhomogénéités des couches superficielles, à des degrés très divers selon la fréquence et la configuration géométrique de mesure. Dans certains cas, il peut traduire principalement le contraste d'impédance eau-fond et est alors directement exploitable, mais avec une portée d'interprétation limitée. Par ailleurs, la mesure d'un niveau absolu de signal implique un étalonnage précis et régulier du sondeur, opération à laquelle bien peu de matériels opérationnels sont soumis, hormis ceux utilisés par des équipes scientifiques (et encore...). Sans prétendre à la détermination du niveau absolu, on peut de manière plus réaliste utiliser les variations relatives du niveau réfléchi moyen sur une zone donnée; ainsi De Moustier [5] a montré la possibilité de détection de nappes locales de nodules de manganèse sur un fond sédimentaire à partir de variations du niveau moyen réfléchi dans un faisceau vertical étroit à $12 \mathrm{kHz}$.

Au delà de la mesure du niveau absolu, Stanton \& Clay [6] ont proposé une détermination des caractéristiques du fond à partir de la.loi de distribution des amplitudes (relatives) reçues: le signal étant décomposé entre une partie cohérente spéculaire et une partie incohérente diffusée par le micro-relief, la mesure du rapport des deux composantes à partir de la distribution des amplitudes (obéissant sous cette hypothèse à une loi de Rice tendant à la limite vers une loi de Rayleigh) permet de remonter à la valeur due la rugosité. Pratiquement cette méthode n'est employable que pour des configurations telles que la partie cohérente du signal reste notable, c'est à dire à des fréquences assez basses. Elle a été testée dans quelques expérimentations $[6,7]$, mais ne semble pas avoir donné naissance à des développements pratiques généralisés.

Une méthode originale de caractérisation de la nature du fond a été proposée par une équipe angloirlandaise (Burns et al.[8]). La méthode consiste à comparer les niveaux relatifs des premiers et second échos réverbérés; le second écho implique deux diffusions par le fond et une par la surface. Cette méthode n'a pas de véritable justification théorique, mais donne des tendances exploitables quant à la relation entre la nature du fond et la structure des deux échos. Un système donné, après apprentissage sur un certain nombre de cas, est alors capable de reconnaître différents types de fonds avec une fiabilité suffisante. Ce dispositif, simple dans son principe et facilement adaptable sur n'importe quel sondeur, a été commercialisé et semble connaître un certain succès.
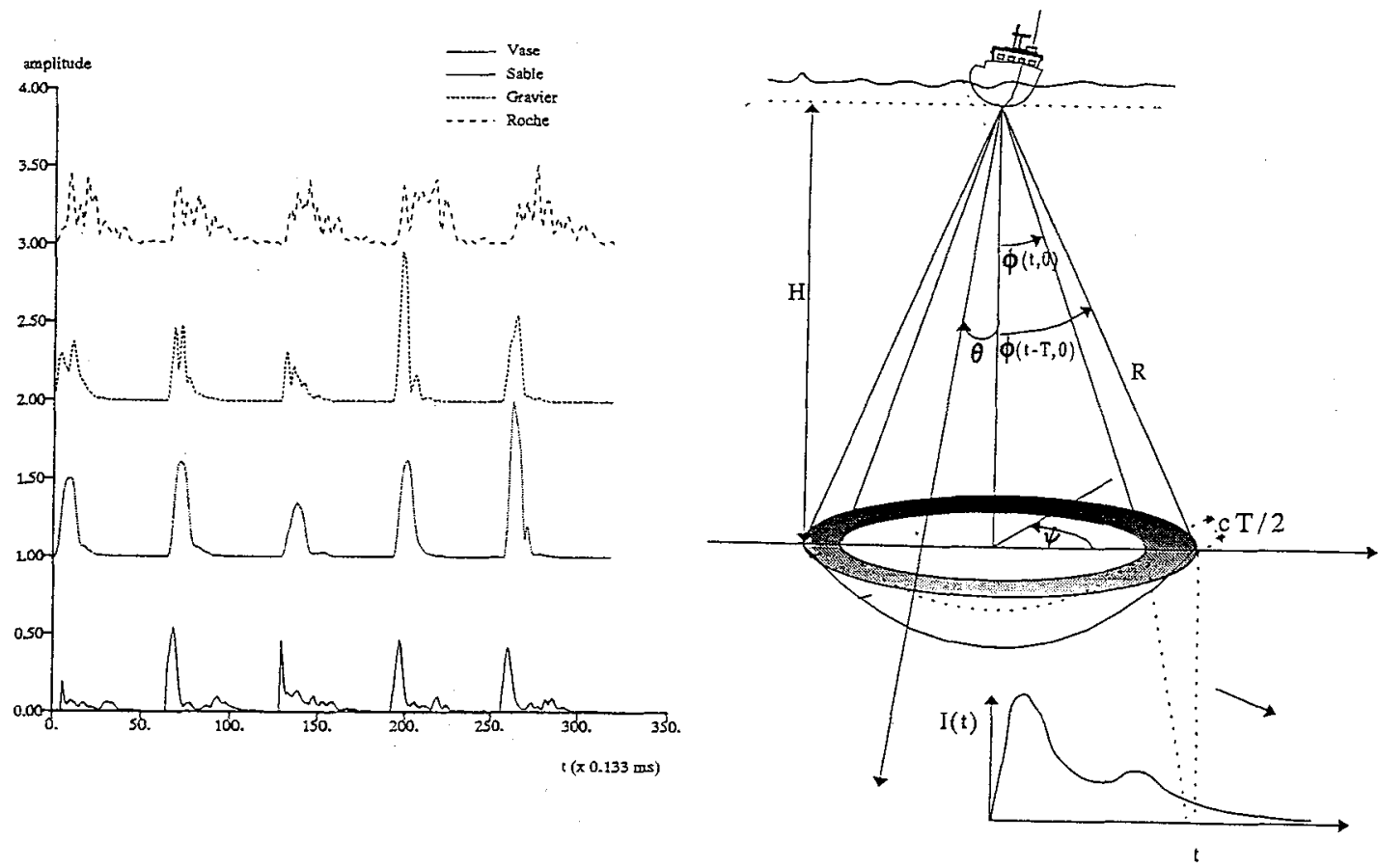

Fig.3. Signaux temporels (à g.) de sondeur monofaisceau rétrodiffusés par divers types de fonds (de $h$. en b.: roche, gravier, sable, vase) et modèle (à dr.) de constitution de cette structure temporelle (Hauteur d'eau de $3000 \mathrm{~m}$, signal de $10 \mathrm{~ms}$ ) 
Plus récemment, Pouliquen \& Lurton [9] ont proposé une méthode d'identification des fonds pour sondeurs monofaisceaux basée sur l'analyse de la structure temporelle des signaux réverbérés. Le principe est que dans un sondeur monofaisceau, l'impulsion utilisée étant très courte et le faisceau émis assez large, le signal temporel effectue un balayage de la zone insonifiée sur le fond, et est donc rétrodiffusé avec des angles variant au cours du temps (fig.3). Le signal temporel est donc lié directement à la réponse angulaire de la rétrodiffusion autour de la verticale; cette dernière étant bien caractéristique du type de fond et ce secteur angulaire étant le plus riche d'information, on peut caractériser la nature du fond à partir du signal temporel. Pratiquement le signal temporel reçu est comparé à des réponses types calculées pour un certain nombre de fonds et de hauteurs d'eau types. La qualité des résultats obtenus par cette méthode est satisfaisante, et a permis son implantation sur un sondeur industrialisé.

\section{UTHLISATION DES SONARS LATERAUX}

Deux approches ont émergé pour l'analyse des données de sonars latéraux en vue de l'identification des fonds marins: l'analyse de la texture des "images acoustiques" obtenues dans la couverture de la zone, et l'analyse spectrale des signaux temporels rétrodiffusés élémentaires. L'approche d'analyse de texture d'images sort du strict cadre des méthodes acoustiques. Elle consiste à appliquer à l'"image-sonar", obtenue après balayage d'une zone par un sonar latéral, des techniques de segmentation et de reconnaissance de texture maintenant classiques en traitement d'images. Des travaux intéressants dans ce domaine ont été menés en France depuis plusieurs années $[10,11]$, à partir d'images de très haute définition obtenues avec des sonars latéraux chasse-mines.

L'analyse spectrale des signaux, proposée par Pace \& Gao [12], consiste à étudier la forme du spectre fréquentiel obtenu à partir du signal temporel rétrodiffusé par le fond. Ce signal temporel étant lié à la propagation le long d'une surface irrégulière, il est en effet raisonnable de penser que son contenu informatif est significatif quant aux caractéristiques moyennes du micro-relief. La méthode consiste simplement, après sélection et conditionnement de l'enveloppe d'une tranche de signal, à en calculer la densité spectrale de puissance, et après moyennage sur plusieurs signaux successifs et compression logarithmique du résultat, à en extraire un petit nombre de paramètres pouvant servir à la classification. Pace \& Gao ont proposé l'utilisation de deux ou trois paramètres traduisant les parts relatives des portions basses et hautes du spectre, mais d'autres analyses sont certainement possibles. Appliquée à l'étude des signaux de sonars latéraux obtenus sur un nombre significatif de zones sédimentaires, ces paramètres permettent une classification avec des résultats vraiment prometteurs. Il est à noter que ces auteurs ont aussi exploré, avec moins de succès, les possibilités d'utiliser pour la classification les paramètres de la loi de distribution statistique des amplitudes d'une part, et les caractéristiques cepstrales d'autre part.

\section{UTILISATION DES SONDEURS MULTIFAISCEAUX}

Les sondeurs multifaisceaux de nouvelle génération ont virtuellement les potentialités cumulées des monofaisceaux et des sonars latéraux; par exemple De Moustier [7] a appliqué à des données de multifaisceaux la méthode de Clay [6] d'analyse des fluctuations de niveaux dans le faisceau vertical. Toutefois, une application plus intéressante de ces systèmes en caractérisation des fonds est originale, et issue de leur structure même: ils permettent en effet de mesurer directement la dépendance angulaire du signal rétrodiffusé, et donc, après corrections convenables, de l'indice de réverbération. Cette méthode a été proposée par De Moustier [7,13] à partir de l'analyse de signaux du sondeur "Sea-Beam". L'intérêt de cette approche est évident, bien que les résultats de De Moustier aient souffert des limitations de performances du sondeur utilisé. Il est également possible d'appliquer aux sondeurs multifaisceaux les méthodes définies pour les sonars latéraux, d'analyse de spectre ou de texture d'images. Un consortium d'équipes de recherches nordiques réunies dans le projet ESMAC autour du constructeur norvégien Simrad travaille dans ce domaine, dans la cas de l'utilisation des sondeurs multifaisceaux en petits fonds [14]. Les premiers résultats récemment présentés laissent apparaître des performances comparables à celles des sonars latéraux, ce qui est raisonnable du fait de la similitude des performances de ces types de système en terme d'ouverture angulaire, de fréquence et de résolution temporelle. Il n'est par contre pas évident que ces méthodes soient applicables efficacement aux données de sondeurs grands fonds à basse fréquence, pour lesquels la surface rétrodiffusante instantanée sur le fond est beaucoup plus importante que les dimensions des structures caractéristiques du micro-relief. 

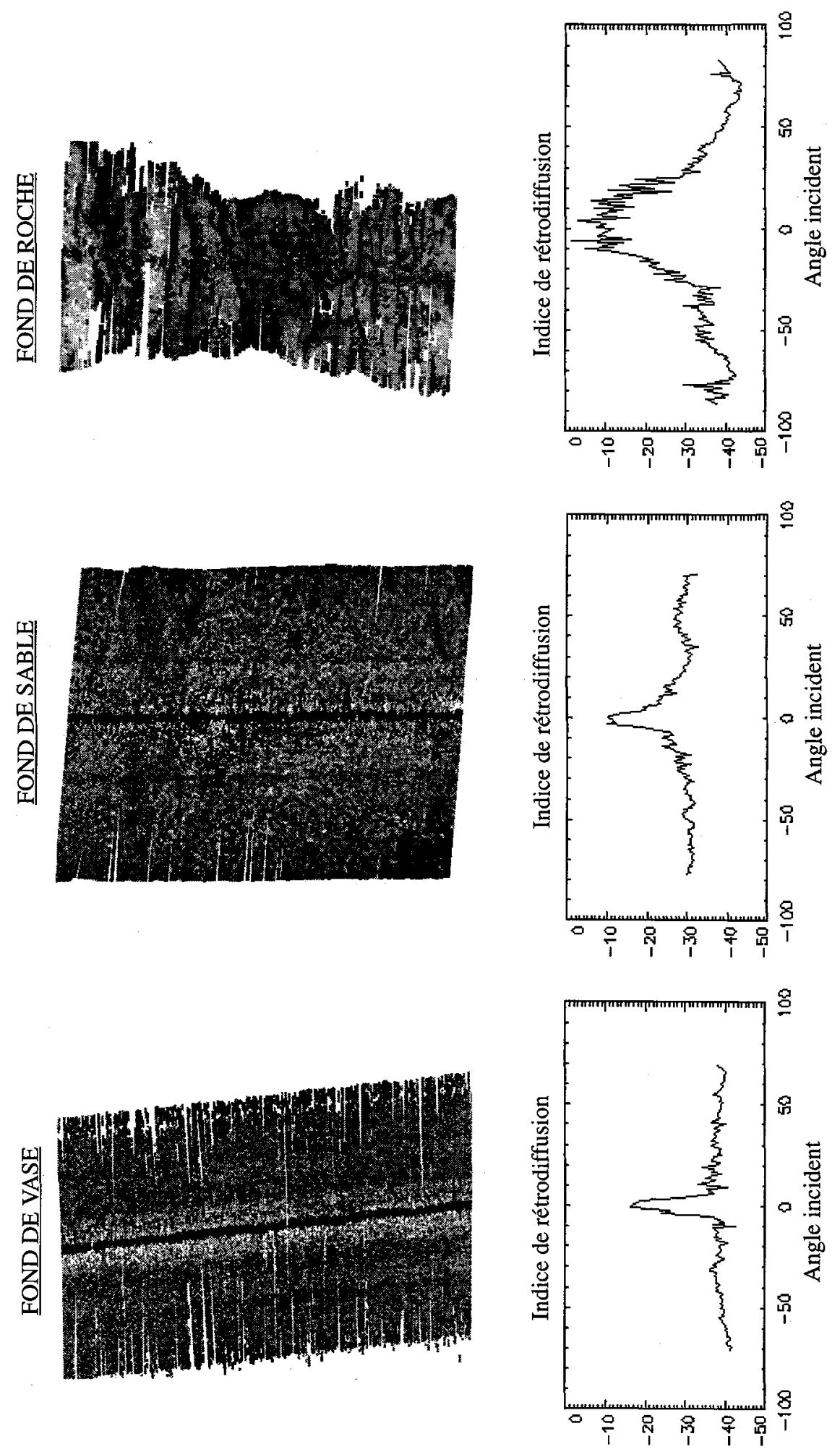

Fig.4. Images acoustiques de sondeur multifaisceaux et indices angulaires de rétrodiffusion pour trois types de fond différents (vase, sable et roche) 
L'IFREMER s'intéresse actuellement à l'exploitation des signaux basse fréquence $(13 \mathrm{kHz}) \mathrm{des}$ sondeurs multifaisceaux grands fonds de nouvelle génération, pour lesquels il n'existe pas encore de résultats d'analyse systématique des données en terme de caractérisation des fonds. Nous avons repris dans un premier temps la méthode de mesure angulaire proposée par De Moustier pour le traitement de données obtenues avec le sondeur multifaisceaux grands fonds EM12D de "L'Atalante". Pour chacun des 162 faisceaux de ce sondeur, espacés d'environ $1^{\circ}$, le système calcule pour chaque signal reçu le niveau d'énergie réverbérée, par correction des pertes de propagation et de la taille de la surface insonifiée instantanément sur le fond. Nous avons été amenés à compenser ces corrections par d'autres formules plus précises d'une part, et surtout à prendre en compte des phénomènes supplémentaires:

- l'angle d'arrivée des signaux sur le fond peut être notablement différent de celui mesuré sur l'antenne, du fait de la réfraction des ondes sonores par le profil profond de célérité, surtout aux incidences les plus fortes;

- l'angle d'attaque du fond par le signal dépend par ailleurs de la pente locale du fond: il faut donc corriger l'angle calculé du signal arrivant sur le fond par une estimation de la pente locale, issue d'un "Modèle Numérique de Terrain" obtenu à partir des données de bathymétrie.

On notera les limitations inhérentes à cette approche:

- le fond est supposé homogène sur toute la plage analysée, qui doit inclure une partie significative du secteur angulaire, donc de préférence autour de la verticale,

- l'analyse doit être faite après détermination du relief local de la zone, ce qui exclut pratiquement son utilisation en temps réel: la détermination ne peut être faite qu'en même temps que la constitution de la carte bathymétrique.

On présente en fig. 4 les résultats obtenus sur trois zones sédimentaires différentes. La première zone correspond à un fond d'argile (Nouvelle Calédonie, hauteur d'eau $4000 \mathrm{~m}$ ), la seconde à un fond de sables grossiers (Golfe de Guinée, hauteur d'eau $2000 \mathrm{~m}$ ), la troisième à un fond rocheux (Dorsale Atlantique, hauteur d'eau $3000 \mathrm{~m}$ ). Pour chacune de ces trois zones, on présente:

- un exemple des images acoustiques obtenues, sur lesquelles on peut déja noter visuellement des différences de texture assez nettes,

- la mesure (moyennée sur la zone présentée) de la variation de l'indice de rétrodiffusion avec l'angle d'incidence sur le fond, corrigé des pentes locales du relief.

On constate que les résultats présentés permettent de différencier nettement les trois zones: le fond d'argile correspond à un indice de rétrodiffusion très pointu autour de la verticale, et à des valeurs assez faibles en latéral (de l'ordre de $-40 \mathrm{~dB}$ ); le fond de sable a un indice plus étalé autour de la verticale, et des valeurs assez élevées aux incidences latérales (environ $-30 \mathrm{~dB}$ ); le fond de roche, pour lequel les corrections de pentes locales étaient très notables, correspond à un indice très étalé en fonction de l'incidence. Il est bien évident que ces résultats correspondent à des configurations assez faciles dans la mesure où les zones traitées étaient homogènes, et où le contraste entre les types de fonds rencontrés sur les trois zones est très important. Toutefois, les tendances sont assez nettes pour laisser augurer favorablement des potentialités de méthodes d'identification automatisés basées sur de telles mesures.

\section{CONCLUSIONS ET PERSPECTIVES}

Bien que des avancées significatives aient eu lieu au cours des dernières années et que plusieurs approches prometteuses aient été identifiées, on est encore loin d'une détermination automatisée de la nature des fonds à partir des systèmes de bathymétrie-imagerie courants, et donc de relevés rapides et fiables de cartes de cette nature.

Les sondeurs monofaisceaux ne disposent que de peu d'information exploitable, n'effectuent que des mesures très locales de par leur principe mème, et sont très sensibles aux mouvements du porteur (lorsque ceux-ci ne sont pas compensés) qui affectent sévèrement la structure des signaux rétrodiffusés. Il est peu probable que des progrès décisifs puissent être obtenus dans l'utilisation de tels systèmes dans leur conception actuelle; les deux dispositifs décrits ci-dessus [8,9] restent limités dans leurs possibilités. Peut être pourrait-on attendre de nouvelles approches à partir de l'analyse des données obtenues avec des sondeurs à bande large utilisant des signaux modulés, mais l'utilisation de tels systèmes est très peu courante

Avec les sonars latéraux, le problème s'est nettement déplacé sur le terrain du traitement d'image, à base de méthodes de segmentation et d'analyse de textures; ceci est tout à fait logique dans la mesure où les 
performances en résolution de ces sytèmes permettent d'assimiler directement le relief diffuseur, le signal rétrodiffusé et limage constituée. II est probable que des progrès continueront d'être obtenus dans ce domaine, profitant de la dynamique de l'évolution des techniques de traitement d'images. En regard, l'intérêt des techniques d'analyse spectrale des signaux rétrodiffusés apparaît finalement moindre: en effet la modulation de la structure des signaux par le relief du fond est tout aussi bien traduite par la texture de l'image obtenue, alors que les techniques de segmentation, essentielles dans la constitution de cartes, ne lui sont pas applicables, au moins dans l'approche actuelle qui traite les signaux successivement; le principal mérite de cette méthode est d'effectuer l'opération d'identification en temps réel dès réception du signal, ce qui peut s'avérer intéressant pour certaines applications.

Les sondeurs multifaisceaux permettront certainement à terme d'obtenir les résultats les plus intéressants. Leurs principaux avantages sont:

- de permettre des mesures objectives de l'indice de réverbération du fond sous une grande variété d'incidences,

- de pouvoir évaluer (et compenser?) l'influence du relief local sur les variations de cette réflectivité telle qu'elle est traduite par les images acoustiques,

- d'obtenir par ailleurs des images comparables à celles des sonars latéraux, du moins en hautes fréquences.

L'identification automatisée des fonds par sondeurs multifaisceaux devra donc mettre en oeuvre des solutions mixtes, faisant intervenir successivement des corrections des niveaux rétrodiffusés par la compensation de l'influence du relief mesuré pour arriver à une image indépendante des effets de topographie, des techniques de segmentation des images corrigées pour définir des zones homogènes, et des opérations d'identification à partir soit de l'analyse de texture d'image lorsque cette dernière est significative (en hautes fréquences) ou dans le cas contraire à partir des niveaux rétrodiffusés, voire des spectres de signaux si ceux-ci s'avèrent être exploitables.

Enfin il convient de garder présent à l'esprit que seuls les moyens d'investigation géotechniques permettent d'arriver à une connaissance précise des caractéristiques du fond, et que les méthodes acoustiques ne sont de toutes les manières que des outils de première approche; et donc de rester modestement réalistes dans ce que l'on peut en attendre...

\section{Remerciements}

L'auteur remercie vivement Eric POULIQUEN, Samantha DUGELAY et Jean-Marie AUGUSTIN pour avoir utilisé les résultats de leurs travaux et pour leur aide dans la préparation de ce papier.

\section{Références:}

[1] Brehhovskikh L. \& Lysanov Yu, "Fundamentals of Ocean Acoustics", Springer-Verlag (1982)

[2]Gensane M. "A statistical study of acoustic signals backscattered from the sea bottom", IEEE Journal of Oceanic Engineering vol.14 $\mathrm{N}^{\circ} 1,1989$

[3]Gensane M. \& Tarayre H. "Tests of sea-bottom discrimination with a parametric array", Acoustic Letters Vol.16 No5, 1992

[4] Pautot G. \& Bellaiche G. "Apport de la fonction imagerie acoustique du sondew Simrad EM12D de l'Atalante sur la connaissance de la nature des fonds océaniques: prolongement du canyon du Var à l'Ouest de la Corse", C.R.Acad.Sci.Paris t. 317 série II pp.663-670, 1993

[5] De Moustier Chr., "Inference of Manganese nodule coverage from Sea Beam acoustic backscattering data", Geophysics vol.50, N०6, pp.989-1001, 1985

[6] Stanton Th. \& Clay C.S., "Sonar echo statistics as a remote-sensing tool: volume and sea-floor", IEEE Journal of Oceanic Engineering Vol.11 $\mathrm{N}^{\circ} 1$ pp.79-95, 1986

[7] De Moustier Chr., "Beyond bathymetry: mapping acoustic backscattering from the deep sea-floor with Sea Beam", JASA 79(2) pp 316-331, 1986

[8] Burns D.R. et al., "Rapid and convenient acoustic sea-bed discrimination for fisheries applications", Proc.I.O.A. vol.11, Part 3, 1989

[9] Pouliquen E. \& Lurton X.,"Identification de la nature du fond de la mer à l'aide de signaux d'échos-sondeurs: I. Modélisation d'échos réverbérés par le fond. II. Méthode d'identification et résultats expérimentaux", Acta Acustica, à paraitre, 1994

[10] Jan D. \& Minot J.,"Le traitement d'image en sonar latéral", L'Onde Electrique vol.69 N³, 1989

[11] Le Gall J., "Analysis and simulation of side scan sonar image texture", Proc.I.O.A. vol 15 Part 2, 1993

[12] Pace N.G. \& Gao H. "Swathe seabed classification", IEEE Journal of Oceanic Engineering Vol 13 N² pp 83-90, 1988

[13] De Moustier Chr. "Angular dependence of 12-kHz seafloor acoustic backscatter", IASA 90(1) pp 522-531, 1991

[14] Kristensen J.H. "ESMAC, a Nordic research program for mapping and characterization of the seafloor", Proc.I.O.A.

Vol.14 Pt2 pp 131-140, 1993 